\title{
LISTERIOSIS IN WESTERN SYDNEY
}

$\mathbf{R}$ esponding to identified clusters of disease is a continuing responsibility for Public Health Units. The type of response is dictated by the type of disease cluster, the size of the cluster, public expectations and political constraints. This report describes an investigation of a cluster of cases of listeriosis in western Sydney and discusses some relevant statistical and epidemiological issues in assessing disease clusters.

\section{BACKGROUND}

On April 24, 1991, the Institute of Clinical Pathology and Medical Research (ICPMR) at Westmead Hospital notified the Infectious Diseases section of the Epidemiology Branch of the NSW Health Department of four cases of confirmed Listeria monocytogenes it had identified.

\section{SUBJECTS}

\section{Case summaries}

1. 66-year-old male.

Frequently hospitalised over past five months with chronic alcoholic liver disease and oesophageal varices. Fevers, nausea and diarrhoea. Cerebellar abscess. Blood cultures positive for $L$. monocytogenes. Lives in western Sydney. Onset 31/3/91?

2. 28-year-old male.

Systemic Lupus Erythematosis recently diagnosed after prolonged illness. More recently fevers, nausea and vomiting. Cultures positive for $L$. monocytogenes. Lives in the central-west of NSW. Onset 2/4/91?

3. 3-year-old female.

Previously well 3-year-old with no other major illnesses. Cultures positive for $L$. monocytogenes following five-day history of fevers, headaches, vomiting and diarrhoea. Lives in western Sydney. Onset 11/4/91.

\section{Female neonate.}

Daughter of a refugee family living in a hostel in southwestern Sydney. Mother well throughout pregnancy. Foetal distress noted at 31 weeks gestation. Blood cultures positive for $L$. monocytogenes. Onset 18/4/91?

\section{Methods}

\section{Case interviews}

Case one and two were interviewed at Westmead Hospital by Dr Wayne Smith. Case three (family) was interviewed at Fairfield Hospital by Dr Jeremy McAnulty. Case four (family) was interviewed at Mt Druitt Hospital by Dr Chris Nielsen. All interviews were done using a specially designed standardised structured questionnaire.

\section{Statistical analysis}

All statistical calculations were done using the SPIDA ${ }^{1}$ statistical package.

\section{RESULTS}

No specific foodstuffs or brands of foods were identified as a common potential source of infection. None of the cases had knowingly consumed any unpasteurised dairy products. No dairy product or vegetable types were common to all cases. Two cases had exposure to domestic animals.

There was no apparent potential source of infection common to all cases. No specific exposure can be implicated in any single case.

\section{DISCUSSION}

Statistical assessment of whether this cluster constitutes an epidemic.

Case definition

A case is defined as laboratory-confirmed listeriosis requiring hospitalisation.

\section{Incidence}

From Benenson, the expected incidence of illness requiring hospitalisation is $1: 150,000$ population per annum.

\section{Population base}

The source population for these cases is approximately 1.8 million, as the cases originated in the three health areas: Western Sydney/Wentworth; Central-Western NSW and South Western Sydney. The expected number of cases in this area per year is 12 . The expected number of cases over three weeks is 0.69 .

The observed number of cases was four over a period of three weeks. Using the Poisson distribution, the probability of this number of cases occurring by chance in this particular three-week period is less than $0.006^{1}$ (Figure 3).

Therefore, four cases occurring in this population over this three-week period satisfies the statistical definition of an epidemic. If three cases had occurred during this timeframe, the result would still be significant $(p<.05)$, but not if two cases had occurred. As seen from Figure 3 , if these four cases had occurred over a time period up to five weeks and five days, the result would still be significant $(\mathrm{p}<.05)$.

\section{Precautions in interpreting this result}

\section{Incubation period}

Because of the large variance in potential incubation period for listeriosis (three days to 70 days), these four cases presenting in this three-week period may have arisen from incident infection over a 13-week period (if 70-day incubation before first case and three-day incubation before last case).

\section{Error in background incidence rate}

Using incidence rates for listeriosis requiring hospitalisation from another region or country is a potentially large source of bias. Figure 3 shows the effect of varying the incidence rate on the probability of a false-positive epidemic.

\section{Statistical issues in defining timeframes}

There are 344 three-week periods in a year. Given that there are six chances in 1000 that four cases of listeriosis requiring hospitalisation can occur purely by chance in a three-week period, there is a:

$$
\begin{aligned}
&(0.994)^{344}= 12.6 \text { per cent probability of AVOIDING a cluster } \\
& \text { of four cases in a three-week period in a year. }
\end{aligned}
$$

That is, there is an 87.4 per cent chance that a cluster of four cases will occur in one of the 344 three-week periods in a year.

\section{Whether to do special studies?}

Case-control studies to determine statistically significant associations between exposure variables and outcome in outbreaks such as this have been done with results providing direction for interventions $s^{3}, 4$. However, such casecontrol studies require at least five cases and high relative risks (about 20 times for five cases) to make them worthwhile. In this case none of these requirements is met, so no special study is justified. 


\section{Listeriosis in western Sydney}

- Confinued from page 733

\section{FIGURE 3}

Probability of a type 1 error (false positive), using the Poisson distribution, of four and five cases of listeriosis requiring hospitalisation occurring over various timeframes using different background incidence rates.

A. FOUR CASES

\begin{tabular}{|lccr|}
\hline & \multicolumn{3}{c|}{ Background Incidence } \\
Time period & $1: 100,000$ & $1: 150,000$ & $1: 200,000$ \\
\cline { 2 - 4 } 3 weeks & $p=.021$ & .006 & .002 \\
4 weeks & $p=.051$ & .015 & .006 \\
5 weeks & $p=.098$ & .03 & .012 \\
6 weeks & $p=.157$ & .052 & .021 \\
\hline B. FIVE CASES & \multicolumn{3}{c}{} \\
\hline & \multicolumn{3}{c|}{ Background Incidence } \\
Time period & $1: 100,000$ & $1: 150,000$ & $1: 200,000$ \\
\cline { 2 - 4 } 3 weeks & $p=.004$ & .0007 & .0002 \\
4 weeks & $p=.014$ & .003 & .0007 \\
5 weeks & $p=.032$ & .007 & .002 \\
6 weeks & $p=.06$ & .014 & .004 \\
\hline
\end{tabular}

\section{SUMMARY AND RECOMMENDATIONS}

Four cases of listeriosis from three Health Areas over a period of three weeks were reported to the Infectious Diseases section of the Epidemiology Branch. This incidence rate represents a statistically significant epidemic for this particular three-week period, if a usual incidence of infection requiring hospitalisation is assumed to be the same in Australia as in the United States.

These points are relevant in considering recommendations:

1. Investigation of the cases reveals no common source of infection, and no particular source for each case.

2. Two of the cases were in immunocompromised patients, and in only one, a 3-year-old girl, were there no predisposing factors.
3. Overall, this cluster of cases of listeriosis fits the classical picture of only particular groups of individuals (neonates, immunosuppressed persons) being at risk from Listeria monocytogenes, an agent common in many foods.

4. Benenson ${ }^{1}$ recommends no concurrent disinfection, quarantine or immunisation of contacts.

5. There is an almost 90 per cent chance that this 'epidemic' will occur in a three-week period in any given year.

6. No special study can be justified.

Taking these points into account, the most appropriate recommendation is to continue surveillance and investigate further clusters, but not to undertake any more formal study, e.g. case-control study, in this case. To this end, pathology services should be alert to the importance of reporting cases of listeriosis. This will require some reinforcement of existing recommendations and feedback from investigations such as for this cluster of four cases, including summaries of what results and actions are possible from such investigations. In this case, to illustrate some possibilities for formal feedback, the Western Sector Public Health Unit has presented the results of this investigation to the microbiological staff at the ICPMR at Westmead Hospital. This presentation included:

\section{investigation methods}

possible further study designs

public health consequences

Health Department public health powers

statistical considerations for defining infectious disease epidemics and

- some discussion of the usefulness of investigating clusters of disease.

Wayne Smith, Public Health Officer Western Sector Public Health Unit

1. Gebski V et al. Statistical Package for Interactive Data Analysis (SPIDA) Version 5.25, 1989.

2. Benenson AS. Control of Communicable Diseases in Man 15th Ed. APHA, Washington DC, 1990.

3. Limnan MJ et al. Epidemic Listeriosis Associated with Mexican-Style Cheese. N Eng J Med, Vol. 319 No. 13, 1988.

4. Schlech WF et al. Epidemic Listeriosis: evidence for transmission by food. $N$ Eng J Med 1983; 308:203-6.

5. Neutra RR. Counterpoint from a Cluster Buster. Am J Epidemio $1990 ; 132: 1-8$

\section{CHILD AND FAMILY HEALTH CONFERENCE}

D $r$ David Hall, who chaired a British working party that produced the radical report Health for All Children, which suggested a more stringent approach to child health surveillance and screening, will be a key speaker at a conference next month at the University of Newcastle. The fourth annual NSW Community Child and Family Health Conference, from January 29 to 31 , is to be opened by the Minister for Health and Community Services, Mr John Hannaford.

Other key speakers will include the Director of the National Health Strategy, Jenny Macklin, who will discuss some of the implications for child and family health services of the national review; the Chief Nursing Officer in NSW, Judith Meppem; the Director of the
Western Australia Research Institute for Child Health, Professor Fiona Stanley; and the Professor of Sociology at the University of Newcastle, Lois Bryson, who will give the closing address with her provocative paper And after all this, who really benefits?

Themes at the conference will include infectious diseases, rural child safety, early intervention and a new look at delinquency. Workshops will examine the use of computers in effective child health service management, domestic violence, alcohol and drug abuse, providing services to mobile families and working with toddlers.

Brochures are available from the Central Coast AHS Child and Family Health Service (telephone 043 203618 ) or the Child, Adolescent and Family Health Service in the Hunter (telephone 04969 4955). 